\title{
Enfants orphelins, enfants séparés, enfants élevés
}

Gardes et apprentissages des mineurs d'âge à Orléans au XV $\mathrm{XV}^{\mathrm{e}}$ siècle

Françoise Michaud-Fréjaville

\section{(2) OpenEdition \\ Journals}

Édition électronique

URL : https://journals.openedition.org/crm/720

DOI : $10.4000 / \mathrm{crm} .720$

ISSN : 1955-2424

Éditeur

Honoré Champion

\section{Édition imprimée}

Date de publication : 1 juin 2005

Pagination : 41-48

ISSN : $1272-9752$

Référence électronique

Françoise Michaud-Fréjaville, "Enfants orphelins, enfants séparés, enfants élevés ", Cahiers de recherches médiévales [En ligne], 12 spécial | 2005, mis en ligne le 28 juin 2008, consulté le 15 décembre 2022. URL : http://journals.openedition.org/crm/720 ; DOl : https://doi.org/10.4000/crm 720 


\title{
risM
}

\author{
$-4-$ \\ Enfants orphelins, enfants séparés, enfants élevés : \\ Gardes et apprentissages des mineurs d'âge à Orléans \\ au $X V^{\mathrm{e}}$ siècle*
}

«L'en otroie tant seulement a ces personnes a avoer orfenins a cels qui par nature ou par debonereté avoent, et de toz autres l'en doit l'en deffendre. Emprés l'en doit voir de quel vie est celi qui veaut avoir l'orfenin et fere de sa mesnie; emprés de quel age cil set qui l'orfenin veaut avoir, por [estimer] s'il doie plus entendre a faire enfans a sei, que a penser de cels qu'il a pris de mesnie $»^{1}$.

Dans sa crudité juridique, ce recueil de droit ligérien du XIII ${ }^{\mathrm{e}}$ siècle envisage avec un sain réalisme, hérité du Digeste, la question difficile du placement des orphelins. On notera avec intérêt que la bonté, le dévouement sont mis à égalité avec la nature, c'est-à-dire le droit naturel des parents pour justifier le choix de tel ou tel tuteur. C'est ensuite seulement que vient la considération de la possibilité matérielle d'assumer la charge de l'accueil à domicile (dans la mesnie...) ; en dernier est envisagée la surcharge qui pèserait sur un nourricier déjà pourvu de famille ou susceptible d'en avoir une. Le cœur l'emporterait-il donc sur la raison?

«Avouer» des orphelins, c'est en avoir le bail, la garde. L'ancienne coutume d'Orléans prévoyait que le parent survivant assumait cette garde, du moins pour la mère tant qu'elle n'était pas remariée ${ }^{2}$, mais pouvait refuser l'administration de leurs biens et ne garder que «le droit de gouverner leurs personnes ${ }^{3} »$. À défaut des pères ou mères, les tuteurs étaient choisis par les proches parents et voisins. La tutelle durait jusqu'à douze ans pour les filles et quatorze ans pour les garçons ${ }^{4}$.

Dans un certain nombre de cas, les tuteurs ont passé devant notaire des actes organisant la garde des enfants, ces accords sont désignés sous le nom de «bail d'enfant » ou de «prise d'enfant ${ }^{5}$ », mais on les rencontre aussi sous la mention plus ambiguë de «louage d'enfant» et, nous avons alors quelques difficultés à les distinguer des contrats d'apprentissage, dont le propos premier devrait être d'enseigner un métier à un jeune et non de lui fournir une famille.

*Sauf indication contraire, les références sont celles des Archives départementales du Loiret.

${ }^{1}$ Li Livre de jostice et de plet, éd. Rapetti, Paris, 1850, (coll. Doc. Inédits), p. 61.

${ }^{2}$ Ancienne coutume d'Orléans, art. 163: «La femme veuve, si elle se remarie, perd la garde de ses enfans, et non le pere lequel, combien qu'il convole en autres nopces, ne perd la garde de sesdits enfans ».

${ }^{3}$ C'est dans l'introduction que donne Pothier du titre IX : «Des enfans qui sont en leurs droits, et de la puissance paternelle» de la Coutume des duché, bailliages et prévôté d'Orléans et ressort d'iceux, Orléans, 1776, t. I, p. 418-419, que l'on trouve de remarquables éléments permettant de comprendre la présence simultanée des tuteurs, des curateurs et du parent survivant.

${ }^{4}$ Anc. cout., art. 162.

53 E 10 136, 19 janvier1431 (n. st.) : mention marginale «prise d'enfant».

Cahiers de Recherches Médiévales (XII - XV S.), 12spé, 2005 
Le rôle joué par les orphelins dans le marché du travail urbain m'a paru, depuis plusieurs années déjà, à la fois digne d'intérêt et méconnu ${ }^{6}$. Les confusions fréquentes entre les "prises » et les «louages »d'enfant m'ont amenée à envisager les deux sources conjointement afin de tenter de mieux comprendre ce que pouvaient être, de façon concrète et non pas idéale et théorique, les moyens d'éducation et d'acquisition de connaissances des enfants des groupes sociaux non privilégiés par la naissance, c'est-à-dire l'immense majorité des jeunes. Après avoir déterminé les éléments principaux qui peuvent caractériser le groupe des orphelins de nos textes, nous essaierons de présenter les modalités d'accueil et d'éducation qui, par certains aspects, leur seraient spécifiques.

De quels jeunes s'agit-il ? Je me suis fixée le butoir de 14 ans comme représentatif de «l'enfance»; au delà, les apprentis peuvent contracter sans tiers, ils peuvent être mis en prison s'ils s'enfuient, enfin ils doivent dans de nombreux cas consolider leur contrat en s'obligeant en lieu et place de leurs répondants. Tous les actes ne donnent pas, hélas, les âges des enfants confiés en bail ou installés chez un artisan, les seules mentions «mineur» ou "suffisamment âgé » ont permis, cependant, de classer tel ou telle de part et d'autre de l'âge critique, mais on ne peut prendre en compte dans tous les cas de figure étudiés ceux que ne désigne qu'un simple qualificatif aussi imprécis. La durée des engagements en effet ne se révèle, nous le verrons, en aucune façon en rapport avec l'âge au moment du contrat et ne permet donc pas d'estimation « régressive ${ }^{7}$.

Les dépouillements des divers types d'acte n'ont pas été faits de la même façon. Ma recherche des apprentis a été systématique et, sans être exhaustive encore, porte aujourd'hui sur près de 1200 cas $^{8}$. Au départ, je dois avouer avoir fait des confusions entre les baux d'enfant et certains apprentissages parce que les notaires emploient le même vocabulaire pour un enfant de six ans ou un adolescent de seize, et parfois ne précisent pas avec netteté les rapports entre les différents acteurs du contrat. Un exemple des confusions possibles peut être trouvé dans le cas des petits Trotereau. En 1464, Pierre, 14 ans, fils de feu Jean Trotereau et de Jeanne est placé par son tuteur, deux de ses oncles et «Jehan le Ribault qui a espousé la dite Jehanne sa mère », chez Pierre Cousteulx, tanneur d'Orléans, qui lui fera avoir la franchise du métier ${ }^{9}$. Un an plus tard, et dans un autre registre, nous retrouvons nos Trotereau: Jean, 13 ans, est introduit par ses tuteurs Pierre Cousteulx et Etienne le Clerc, chez un tisserand en drap nommé Jehan le Ribault ${ }^{10}$. Sans le rapprochement de ces deux

${ }^{6} \mathrm{~F}$. Michaud-Fréjaville, «Contrats d'apprentissage en Orléanais, les enfants au travail (13701450) », L'enfant au Moyen Âge, Senéfiance n 9, 1980, p. 63-71.

${ }^{7}$ Autrement dit, il n'est pas possible de soustraire le nombre d'années d'engagement à 14 ans pour les garçons ou 12 ans pour les filles pour trouver l'age au contrat des enfants.

${ }^{8} \mathrm{~F}$. Michaud-Fréjaville, «Bons et loyaux services, les contrats d'apprentissage en Orléanais (1380-1480)», Les entrées dans la vie, XIII congrès des Historiens Médiévistes, Nancy, 1981, p. 183-208 ; également, "Crise urbaine et apprentissage (1475-1500)», Villes, bonnes villes et capitales, Mélanges en l'honneur de Bernard Chevalier, Tours, 1989, p. 13-24; et "Apprentis et ouvriers vignerons, les contrats à Orléans au $\mathrm{XV}^{\mathrm{e}}$ siècle », Le vigneron, la viticulture et la vinification, Flaran 11, 1989, p. 274-285. Articles qui figurent dans le présent recueil.

${ }^{9} 3$ E 10162, 8 juillet 1364. Précisons que c'est l'apprenti qui paiera le droit d'entrée.

${ }^{10} 3$ E 10163,14 octobre 1365. 
pièces, nous ignorerions totalement que tout cela se passe en famille, que les deux enfants sont placés, l'aîné chez un proche, et le second au foyer maternel! Ce cas n'est pas unique et illustre les risques d'appréciations aventureuses, éventualités qu'il faut néanmoins assumer. Ces deux garçons ci-dessus sont restés comptabilisés parmi les apprentis parce que leurs contrats ne se distinguaient en rien des autres. Mais les «baux » d'enfants ont du coup attiré mon attention et ont été repérés avec le plus de rigueur possible, cependant ils n'ont pas fait l'objet d'un dépouillement systématique, mais d'un sondage de cent actes répartis entre plusieurs notaires et étalés au cours du siècle ${ }^{11}$.

Nous présentons donc une population dont les données statistiques sont les suivantes : 993 contrats d'apprentissage à Orléans nous proposent 563 orphelins, dont 231 sont âgés de moins de quatorze ans, (17 filles et 214 garçons). À côté, nous avons donc une centaine de baux d'enfants qui totalisent 141 individus orphelins, soit 77 filles et 64 garçons.

On constate d'une part, une prédominance absolue des garçons $(92,6 \%$ des occurrences) pour l'entrée chez un patron, et de l'autre, une récupération des filles, moins écrasante mais réelle $(54,6 \%)$, pour l'accueil auprès d'un parent ou d'un tuteur. La disproportion des filles serait d'ailleurs moins grande si de faux contrats d'apprentissage n'avaient dû être reversés parmi les baux d'enfants, venant renforcer l'élément masculin de l'apprentissage ${ }^{12}$.

Quels décès amènent nos enfants dans les registres, toutes catégories confondues? La surmortalité paternelle est écrasante: 62,1\% seraient des orphelins de père, $20,4 \%$ de mère, $11 \%$ apparaissent privés de leurs deux parents, enfin pour $6,5 \%$ des cas, l'impétrant étant représenté par les tuteurs ou des proches et sans indication de filiation, le ou les parents disparus ne peuvent être repérés. Nous aurions donc près des deux tiers de ces jeunes éloignés de l'éducation par l'exemple, acquise auprès de l'adulte mâle du couple conjugal. Ce déséquilibre est, bien enten$\mathrm{du}$, lié à l'exercice de la puissance paternelle : pour que l'engagement pris par une mère ou un tiers soit légal, il faut qu'en place du mineur les contractants signalent que le père ne peut exercer son autorité; en revanche le père, si le patrimoine de l'enfant n'est pas en cause, n'a nul besoin de préciser le décès de sa compagne à moins que le notaire ne veuille le préciser. C'est pourquoi, lorsqu'on considère les seuls baux d'enfants, qui sont plus précis sur la situation familiale, on voit s'accroître la proportion des orphelins de mère qui passe à $34 \%$ des groupes familiaux, et baisser celle des sans père à $58 \%$.

Nous avons donc un ensemble assez particulier, présentant des sujets parfois très jeunes (Jean Pelaut, auquel le bail d'enfant prévoit de laisser « la robe noire de son défunt père », n'a qu'un an et demi ${ }^{13}$ ) et de rangs sociaux fort différents, même si

\footnotetext{
${ }^{11}$ Trente-six registres entre 1388 et 1503 .

${ }^{12}$ Un exemple, entre autres Pierre Martin ( 8 ans) et Jean (5 ans) placés le premier chez son grand-père maternel, le second chez un oncle doivent « apprendre » le métier de vigneron (3 $\mathrm{E}$ 10163,12 février 1303 (n. st.).

${ }^{13} 3$ E 10163, 13 septembre 1465. Trois enfants Pelaut, Blanche, 6 ans, Ragault 4 ans et Jean, sont confiés à leur mère, les deux filles pour 4 ans, le garçon pour 6 ans. La robe noire seraitelle de clergie, comme le suggérait un membre du colloque?
} 
la garde noble, dotée d'une coutume tout-à-fait particulière, n'entre pas dans notre propos $^{14}$.

Les garçons s'y trouvent favorisés par la pratique de l'apprentissage. Si nous reconsidérons l'ensemble des jeunes de moins de 14 ans qui réussissent à trouver une place auprès d'un chef d'atelier ou d'un exploitant agricole, nous ne pouvons que constater la prédominance des orphelins aux âges les plus tendres. Si l'on rapporte les orphelins au nombre total des apprentis par tranche d'âge, il apparaît nettement qu'avant la majorité les deux tiers des places sont occupées par des enfants qui n'auraient peut-être plus ailleurs de milieu familial ${ }^{15}$.

Âge des apprentis à l'établissement du contrat

\begin{tabular}{|l|l|l|l|l|l|l|l|l|l|l|l|l|l|l|}
\hline & ÂES & 2 & 3 & 4 & 5 & 6 & 7 & 8 & 9 & 10 & 11 & 12 & 13 & $\begin{array}{l}14 \\
\text { ans }\end{array}$ \\
\hline I & $\begin{array}{l}\text { Tous } \\
\text { apprentis }\end{array}$ & 1 & 1 & & 4 & 5 & 5 & 18 & 12 & 19 & 18 & 43 & 58 & 119 \\
\hline Il & $\begin{array}{l}\text { Orphelins } \\
\text { / app. }\end{array}$ & 1 & 1 & & 3 & 3 & 5 & 13 & 4 & 15 & 12 & 38 & 31 & 69 \\
\hline $\begin{array}{l}\% \\
(\mathrm{II} / \mathrm{I})\end{array}$ & 100 & 100 & & 75 & 60 & 100 & 72 & 33 & 79 & 66 & 88 & 53 & 58 \\
\hline
\end{tabular}

Nous ne saurions dire si c'est un «avantage» par rapport à ceux qui ont conservé leurs parents, mais de façon nette les patrons donnent la préférence ou se sentent moralement et financièrement plus enclins à ouvrir leurs ateliers à ceux dont les foyers parentaux sont bouleversés par la mort. Ce qui signifie qu'arrivés à l'âge «normal» de l'entrée en apprentissage, après la majorité, bien des orphelins seraient pour partie déjà formés aux pratiques des gens de métier.

Les baux d'enfant, on l'a vu, rattrapent la sous-représentation féminine, confortant le côté domestique et familial, plus intérieur, en quelque sorte, de leur éducation ${ }^{16}$. La différence faite entre les filles et les garçons, les premières gardées à la maison et les seconds envoyés à l'extérieur n'est pas une interprétation abusive des chiffres ; cette disparité est parfois clairement affichée et nous livre une des clefs de nos documents. En 1437, Jean Laduole et Guillaume Commenat tuteurs « ou nom et comme tuteur qu'ils disoient estre » d'Agnès et Catherine Commenat, filles de feu Jean Commenat, confient les fillettes à leur mère Catherine à nourrir «jusqu'à ce qu'elles soient âgées chacune de dix ans ». La veuve devra payer leurs dettes et remettre à chacune en fin de contrat « une coeste, ung coessin, un langeau, deux draps de deux lez, une nappe, ung oreiller, un queuvrechef, et ung coffre ou huche ferment

\footnotetext{
${ }^{14}$ « Au regard de nobles mineurs, ils demeurent dans la garde de père ou mère, ayeul ou ayeule survivant, selon la proximité du degré. Et s'ils n'ont pas de parens en ligne directe, ils chéent en bail de leur prochain parent, idoine et suffisant s'il en veut prendre la charge », Anc. Cout., art. 161 .

${ }^{15}$ On notera par ailleurs dans cette suite de chiffres la tendance bien connue des témoins et autres à se fonder de préférence sur les nombres pairs.

${ }^{16}$ Les deux plus jeunes enfants placés on apprentissage sont cependant des filles auxquelles, outre la fonction de «chambrières », on enseignera le métier de couturière.
} 
de clef », les tuteurs abandonnent à la mère les revenus des héritages des fillettes, à charge pour elle de les entretenir. Le même notaire enregistre à la suite un second contrat par lequel Jean Pardoine, curateur de Perrin Commenat, donne à nourrir celui-ci à Catherine sa mère pendant les deux années à venir. Perrin sera tenu de la servir "en fait de vignerie», recevra sept livres en fin de service, deux draps, un couvre-chef, un oreiller, une nappe, une huche fermant, « et tous les oetiz et abillements de charpentier appartenant au dit feux Jehan Commenat son père », et en outre «sera tenue la dite Catherine de donner pacience au dit Perrin son fils depuis la Saint-Martin d'été jusques à la Saint-Denis ensuivant pour faire lui apprendre, où bon lui semblera apprendre, le mestier de charpentier»; enfin, le garçon aura une partie des vendanges et des tonneaux des vignes de sa mère et ses sœurs sans en payer les façons ${ }^{17}$. Nous comprenons donc la disparité des sex-ratios de nos sources : la succession au métier du père ou la mise en apprentissage sont en effet prévues pour les garçons et non pour les filles. Nous sommes en présence de deux comportements sexués : l'entretien domestique pour les filles, et la véritable éducation celle qui conduit hors des murs de la maison - pour les garçons.

Ces deux textes, d'autre part, présentent ce que les baux d'enfant confirment: dans la majorité des cas, les enfants ne sont pas tout d'abord séparés si l'un des parents survit. Sur les 82 orphelins de père, 57 (70\%) seront accueillis chez leur mère, même si celle-ci est remariée (ce qui lui faisait perdre, rappelons-le, l'automatisme de la garde des enfants); or près d'une veuve sur deux de nos actes a convolé en secondes noces, événement qui a provoqué sans doute le passage devant le notaire. Les pères prennent dans une proportion bien supérieure $(84 \%)$ leurs enfants restés sans mère, ce qui laisse supposer des remariages encore plus nombreux, un homme seul assumant difficilement une garde, et surtout cela peut expliquer la pratique quasi systématique du placement postérieur des garçons chez un patron, mesure allégeant, entre autres, la charge des jeunes appétits masculins dans un second ménage. Finalement, seulement moins du tiers des enfants était transplanté dans un foyer a priori nouveau, et encore faut-il bien voir qu'oncles en particulier, mais aussi frères, tantes, voire plus rarement parrains étaient mis à contribution : 24 enfants ont été placés "à nourrir» dans leur famille et 17 apprentis reçurent leur formation au sein de leur parentèle ${ }^{18}$. La pratique de préserver, dans la mesure du possible, les liens de proximité entre les frères et sœurs me paraît confirmer une certaine prise en compte de la sensibilité enfantine lors des conseils de famille.

Bien sûr, il existait des circonstances où les familles éclataient et en raison desquelles nous assistons à la séparation entre frères et sœurs: ainsi en 1451, Etienne Duran confia Jeanne, 11 ans, comme chambrière à un vigneron, Naudin

${ }^{17} 3$ E 10144, dimanche 10 février 1437 (n. st.). Une même distinction est faite pour les trois enfants Leclerc : Jean ( 5 ans) ira chez son cousin apprendre « le fait de vignerie », mais ira un an à l'école ; Regnault ( 8 ans) sera pris chez son oncle, en même temps que de l'envoyer à l'école on «lui fera apprendre sa créance ». Pour Catherine (10 ans) qui part en compagnie de Regnault rien d'autre n'est prévu que son «nourissement»(3 E 10245, 20 février 1496 (n. st.).

${ }^{18}$ Tous n'accueillent peut-être pas l'enfant ou les enfants comme cet oncle qui le fait «pour Dieu et aumône et sans autre chose des dits tuteurs ni du dit nepveu » (3 E 10127, 30 août 1410). 
Gillebert, et le même jour le frère cadet, Pierre, 8 ans, fut envoyé à Fontenay-sousBagneux-lès-Paris, au service de son frère aîné ${ }^{19}$. Mais il semble bien que la dispersion des enfants se soit en général plutôt faite dans un second temps, au moment où une occasion se présentait, ou, plus logiquement, quand l'enfant à placer avait atteint un âge permettant un véritable contrat d'apprentissage.

Les différences entre filles et garçons pouvaient être également liées aux sacrifices financiers que les tuteurs étaient décidés à faire pour l'éducation des enfants. Des contrats, en petit nombre, aussi bien de prise en charge que d'apprentissage, prévoyaient d'envoyer l'enfant à l'école. Les garçons en semblaient nettement plus dignes que leurs sœurs: une vingtaine de gamins et seulement trois demoiselles purent profiter de l'A, b, c, s'exercer à l'écriture ${ }^{20}$, au besoin aux frais de l'enfant ${ }^{21}$, dût l'apprentissage en être perturbé ${ }^{22}$ et pas seulement quand le patron était Jean Jolis, le libraire de l'Université ${ }^{23}$ ou un licencié en décret $^{24}$. Les frais que les gardes d'enfant supposaient étaient de façon naturelle couverts en partie par les revenus de l'héritage - quand il y en avait un. Les maitres profitaient de cette possibilité en demandant non seulement à faire payer leur peine et les risques de malfaçon ${ }^{25}$, mais également en récupérant les héritages le temps du contrat. Ainsi en prenant Perrin Mulot, le vigneron Thomas de Normandeau lui fera-t-il aussi travailler les deux arpents, dont trois quartiers de vigne, que le tuteur de Perrin lui a confiés, à faire « de bonne façon» et dont le patron touchera le produit ${ }^{26}$. Quant à Pierre Barryere, cordonnier, en raison de «certain petit meuble que [il] dit avoir dudit Pierre [Pierre Adam, l'apprenti, il] le vestira presentement d'une jaquete, un pourpoint, unes chausses, deux chemises, un chapeau... $»^{27}$. Enfin, Jean Hillaire (par ailleurs connu comme un des édiles orléanais) s'assure - pour quatre ans seulement, il est vrai - une augmentation de capital de 50 1.t. en investissant dans ses affaires ce que Jean Bon-

${ }^{19} 3$ E 10 156, 9 décembre 1451 : Manon de Beauvilliers, 9 ans, est séparée de ses quatre frères et sœurs et envoyée chez une tante du côté maternel qui s'engage à lui faire apprendre à coudre (3 E 10131, 21 novembre 1411).

${ }^{20}$ Jean Paugin envoyé par son père chez un potier d'étain, le père demande à ce que l'enfant (12 ans) aille à l'école "jusqu'à ce qu'il sache ecrire se il y est abilhe » (3 E 10153, 29 octobre 1448).

${ }^{21}$ Bastion Caseau, vigneron, enverra son apprenti Jean Angenault «à l'escripture par l'espace de demy an, en paient par le dit alloué son escollage» (3 E 10245, 2 novembre 1495). Les Angenault, il faut le dire, étaient de gros vignerons, que l'on retrouve à de nombreuses reprises dans la clientèle des notaires.

${ }^{22}$ Le patron de Philippon Orry, un vigneron d'Ingré, proche village à l'ouest d'Orléans, ne s'engage que pour une demi-année (3 E 10 235, 6 Janvier 1486 (n. st.). En revanche, Jean des Champs aura droit à un an et demi «de novembre à l'Ascension et un an prochainement venant » (3 E 10211, 11 novembre 1466).

${ }^{23} 3$ E 10132,19 août 1414.

${ }^{24} 3$ E 10137,3 novembre 1434.

${ }^{25} 3$ E 10210, 5 juin 1465, Perrine, veuve de Jean Gauthier, s'engage à rembourser toutes les «pertes et mises et interest qu'il [Louis Morquet, épicier] pourrait avoir [...] par la faulte et coulpe dudit apprentiz et par défaut dudit service non fait ».

${ }^{26} 3 \mathrm{E} 10130,10$ mai 1411.

${ }^{27} 3$ E 10164, 23 janvier 1467 (n. st.). 
nin a eu de la succession de son père, «et dont Jehan Hillaire lui rendra compte avec le retour de XLV 1.t. seulement $»^{28}$. À qui profite l'apprentissage?

Que les conditions morales et matérielles aient pesé sur l'entrée précoce de l'orphelin chez un patron ne me semble donc ne faire aucun doute, cependant il faut noter que l'âge tendre n'avait que peu d'influence sur la longueur prévue de la présence dans le ménage d'accueil. La durée normale se situe entre trois et six ans ( $77 \%$ des contrats, 160 cas), les durées supérieures, qu'on pourrait concevoir en raison de la jeunesse des individus, se réduisent à quarante-deux exemples pour un siècle, ce qui est peu. Surtout les longs laps de temps ne sont pas vraiment en corrélation avec le moment de l'entrée en service. À l'exception des bébés - les deux cas supérieurs à 12 ans concernent des bambins de 3 et 5 ans - les relations âge/durée semblent plutôt aléatoires : à 8 ans, comme à 14 ans, on est accueilli pour 7,8 ou 9 ans. Ici, ont joué des arguments dont aucun texte ne nous donne la teneur. En revanche, les baux d'enfants fixent parfois la durée de la garde en fonction de la majorité, ce qui est normal puisque alors l'héritier rentre en possession de ses biens et reçoit les comptes... en principe. L'acquis principal de son héritage n'était-il pas de lui avoir permis une formation?

$\mathrm{Y}$ avait-il un réel souci d'éducation, de formation, dans le placement des orphelins, soit dans leur famille, soit chez un patron? Le séjour chez un patron est-il une façon déguisée de caser un orphelin, une sorte d' "apprentissage parking ", si j'ose me permettre cet anachronisme? Peu nombreuses, de fait, sont les mentions qui prévoient soit une véritable «introduction» dans un métier, comme pour Perrin Commenat cité plus haut ou Thomas Calla qui sortirait de l'atelier avec sa carde (celle qu'il aura reçue neuve en entrant et ne le sera plus), un demi-écu d'or en poche et l'inscription au métier ${ }^{29}$. Les participations aux confréries de métier sont également bien faibles et n'apparaissent qu'au cours de la seconde moitié du siècle; quand Laurent du Vau paie à la confrérie des serruriers les deux fois 16 sous de ses deux apprentis, Guiot et Jean Macé, il se réserve le droit de les récupérer ${ }^{30}$; néanmoins la confrérie aura pu rendre ses services aux sociétaires.

La qualité réelle du travail proposé aux enfants n'est pas plus précisée pour les orphelins que pour les autres jeunes qui entrent en apprentissage et peu se voient garantir de devenir « experts $»^{31}$.

Les malheurs des temps et les conditions particulières des impétrants font que les clauses de décès apparaissent aussi bien dans les baux - toujours en raison des curatelles et des héritages en indivision-que dans les véritables «louage

${ }^{28} 3$ E 10132,29 janvier 1415 (n. st.).

${ }^{29} 3$ E 10162, 2 juillet 1464. Seront également «introduits », de futurs tisserands en toile et en linge, un boulanger, un menuisier. Cependant le nombre total prévu d'intégration au «métier » n'excède pas la douzaine, c'est peu en un siècle...

${ }^{30} \mathrm{Ils}$ devront les rembourser en fin de service ou « servir à la valleur d'icelle somme par temps suffisant au dit de gens de bien d'icellui mestier» (3 E 10211, 12 décembre 1466). On notera que les deux frères étaient placés chez un patron commun.

${ }^{31}$ Pasquier du Pont garantit pourtant à Raoulant le Pescheur de «lui montrer l'art du dit mestier [de tisserand en drap] en telle maniere que la quatrieme année il sera mestre et le rendra le dit Pasquier en place et l'acquitera de XVI sols par. qui seront deuz en la fin dudit temps à la confraierie des textiers en la paroisse Saint-Pol» (3 E 1064, 1er janvier 1467 (n. st.) ; c'est l'exception qui confirme la règle. 
d'enfants »: quand le jeune Jean Chabaud entre à 8 ans chez le chaussetier Jean Bonnet, il lui remet un petit ménage qui restera à son patron si l'apprenti meurt durant le contrat ${ }^{32}$.

On sait bien que si l'enfant ne se plaît pas dans son nouveau cadre de vie, il peut avoir envie de se sauver. La considération de la faiblesse puérile n'est pas absente des sources, pourtant peu enclines à la tendresse ; pourtant il arrive, et surtout dans les gardes, que la contrepartie du "service» soit la promesse de traiter doucement et justement l'enfant accueilli, sous peine de se le voir enlever avec les sanctions pécuniaires que cela suppose ${ }^{33}$. Le contrat peut donc n'être valable qu'après l'accord de membres de la famille : la mère de Jean Vacher demande une réserve permettant à son propre père de récupérer l'enfant si le grand-père n'apprécie pas le contrat $^{34}$. Le risque demeure pourtant de voir un jour l'enfant disparaître, et les responsables s'engagent donc pour la plupart à ramener le récalcitrant chez son patron. Qu'en advenait-il dans la réalité ?

Bien sûr, je ne saurais conclure que c'était un privilège que de perdre dans sa prime enfance l'un ou l'autre de ses parents, qu'ainsi s'ouvrait la voie à un apprentissage précoce et d'autant plus formateur, et s'acquérait une assurance de démarrer à la majorité avec un petit capital, l'héritage soigneusement préservé par la vigilance familiale $^{35}$. Mais le naturel avec lequel les contrats parlent de la présence à l'école élémentaire, de travail en atelier ou dans les champs, montre que ce sont des activités considérées comme normales pour des enfants. L'intérêt de nos contrats est de présenter ces activités non pas comme une possibilité mais presque comme un droit pour les orphelins et un devoir moral pour ceux qui assument leur garde.

\footnotetext{
${ }^{32}$ « Une coeste, un coessin, ung langeau, une poelle d'arin, une nappe, une toualle, III lb. de fille de chanvre » (3 E 10132, 6 avril 1414 n. st.).

${ }^{33}$ " Se la dite Guillemette est mal gouvernee et qu'elle soit bateue ou chasblee [châtiée] par le dit Guillot ou ses gens autrement qu'il n'appartiendra, il sera au choix dudit [père] ou des amis de la dite fille de la oster et mettre hors du gouvernement et des mains dudit Guillot». Pour être juste, il faut signaler qu'un contrat similaire mais en sens inverse confie la fille de Guillot au père de Guillemette, chassé-croisé dont le sens est pour le moins obscur (3 E 14329, 9 septembre 1412).

${ }^{34}$ «Et s'il advient que Philippon de Rix [...] ayeul du dit [Philippon] ne soit content dudit marché et apprentissage, en ce cas ledit preneur le lui promet rendre » (3 E 10244, 17 avril 1494).

${ }^{35}$ De fait, dans bien des cas l'orphelin s'est vu contraint de vendre « duement autorisé » par ses tuteurs, une part de son héritage pour financer sa propre formation. Ainsi Jean des Champs déjà cité « se aidera de la somme des XII 1. VII s. IIII d. par. appartenant a icellui myneur pour certains biens meubles demourez du décès de son pere et qui ont estes vendus et baillés au dit Jehan des Champs pour ladite somme. Et se aidera le dit Jehan [...] de la revenue des heritages d'icellui myneur »; cependant, à la fin du contrat, Jean, qui est en même temps l'oncle paternel de l'enfant, lui rendra 12 livres et les huit années d'arriérés de 24 s.t. de rente.
} 